\title{
Clinical characteristics of pruritus in patients with bullous pemphigoid: a preliminary questionnaire-based study
}

\author{
Agnieszka Kalinska-Bienias ${ }^{1}$, Emilia Kowalczyk ${ }^{1}$, Pawel Jagielski², Anna Lesniewskaํ․ Aleksandra Komorowska ${ }^{3}$, \\ Cezary Kowalewski ${ }^{1}$, Katarzyna Wozniak ${ }^{1}$
}

${ }^{1}$ Department of Dermatology and Immunodermatology, Medical University of Warsaw, Warsaw, Poland ${ }^{2}$ Human Nutrition Department, Faculty of Health Science, Jagiellonian University Medical College, Krakow, Poland ${ }^{3}$ Department of Dermatology and Venereology, Medical University of Warsaw, Warsaw, Poland

Adv Dermatol Allergol 2020; XXXVII (6): 938-942 DOI: https://doi.org/10.5114/ada.2020.102111

\begin{abstract}
Introduction: Bullous pemphigoid (BP) is the most common autoimmune blistering disease. Although pruritus is a leading symptom in $\mathrm{BP}$, its specific characteristics have not been explored.

Aim: To determine the itch characteristics in newly diagnosed BP patients by using the Questionnaire of Descriptive Assessment of Pruritus and visual analogue scale (VAS) and to correlate itch with disease severity.

Material and methods: The study included 32 patients with BP (mean age: $75.4 \pm 12.2$ years; 20 women, 12 men), who filled in the questionnaire. Bullous Pemphigoid Disease Area Index (BPDAI) was assessed.

Results: In $78.1 \%$ of BP patients pruritus occurred at least once daily. Pruritus was most frequent in the evening and at night and frequently persisted for more than $10 \mathrm{~min}$ in more than a half of patients (long itch episodes). Aggravating factors were sweating (50.0\%), heat (59.4\%) and stress (46.9\%), but the major relieving factor was cold (34.4\%). Itch intensity in BP was assessed as moderate (mean VAS score: 5.8 points) and did not correlate with disease severity (BPDAI). BP patients declared difficulties caused by pruritus: falling asleep (53.1\%) and awakening at night (50\%).

Conclusions: This study provided detailed characteristics in BP patients confirming that pruritus is classified as an important troublesome symptom. Therefore there is a need to search for therapeutic solutions.
\end{abstract}

Key words: bullous pemphigoid, pruritus, visual analogue scale, itch questionnaire.

\section{Introduction}

Bullous pemphigoid (BP) is the most common autoimmune blistering disease characterized by tense blisters and urticarial lesions typically localized on the skin in different areas. It has been shown that BP is accompanied by pruritus. Moreover, pruritus in BP may precede the appearance of blisters and urticarial lesions as the only symptom (a prodromal phase) what poses diagnostic problems [1-3]. The time period from initial pruritus to clinically apparent BP is variable and ranges from several weeks to even 10 years [3, 4]. It has also been shown that pruritus in patients with autoimmune bullous diseases including BP may negatively impact the quality of life (QOL) measured by disease-specific Autoimmune Bullous Disease Quality of Life Questionnaire (ABQOL) [5]. Furthermore, the issue which consistently yielded a negative quality of life in BP was the presence of depression [5]. It is not surprising that the association with major depressive disorders has been well documented in patients with BP $[6,7]$. On the other hand, depression or emotional stress is often the result of pruritus, which was observed in many patients with atopic dermatitis (AD) or psoriasis vulgaris (PV), in which pruritus is a predominant symptom $[8,9]$.

Although it is generally accepted that patients with BP experience pruritus, no detailed analysis and characteristics of itch in BP have been conducted. However, the recent study based on the subjective itch numeric scale has suggested that pruritus in BP is even as intense as in atopic dermatitis [10]. The present study was performed to determine the intensity and qualities of pruritus in BP.

Address for correspondence: Agnieszka Kalińska-Bienias MD, PhD, Department of Dermatology and Immunodermatology, Medical University of Warsaw, 82a Koszykowa St, 02-008 Warsaw, Poland, phone: +48 606618 564, fax: +48 502 21 04, e-mail: agnieszka.kalinska@interia.pl Received: 17.12.2018, accepted: 30.04.2019. 


\section{Aim}

The major goal of the study was to determine the itch characteristics in BP patients based on the patients' reported itch questionnaire and itch visual analogue scale (VAS), and to correlate it with disease severity measured by Bullous Pemphigoid Disease Area Index (BPDAI) [2].

\section{Material and methods}

The study included 32 with BP (mean age: $75.4 \pm 12.2$ years, range: 40-100; 20 women, 12 men). Patients were recruited from 2013 to 2014. The diagnosis in BP was determined based on direct immunofluorescence (DIF) which showed IgG and C3 deposits along the basement membrane zone (BMZ), and indirect immunofluorescence (IIF) which revealed circulating IgG anti-BMZ antibodies. In all BP cases, circulating antibodies reacted with BP 180 NC16a domain in the commercial ELISA kit Mesacup BP180 Test (MBL Co., Nagoya, Japan). The patients with BP were newly-diagnosed, referred to the department to establish the diagnosis of BP. During the study the presence of any other concurrent skin disease that may also cause pruritus such as renal or liver impairment and other acute or chronic diseases was the exclusion criterion. The disease severity was measured by BPDAl. All patients were requested to complete the Questionnaire of Descriptive Assessment of Pruritus authored by Szepietowski and Reich (2010) [11]. The questionnaire consists of 12 questions regarding the details of pruritus such as duration of itch episodes (< or > than $10 \mathrm{~min}$ ), occurrence at different times of the day, aggravating and relieving factors, taking anti-pruritic medications, sleep disturbances caused by itch and taking sleeping medications, effect of pruritus on quality of life. The intensity of itch was assessed by itch VAS in all patients. Based on literature data, the following interpretation criteria were used: $0=$ no itch, $1-<4$ points = mild itch, $\geq 4-<7$ points = moderate itch, $\geq 7-<9$ points $=$ severe itch, and $\geq 9$ points = very severe itch [12]. Consent of the Bioethics Committee of the Medical University of Warsaw was obtained prior to the study. All patients gave their informed consent to participate in the study.

\section{Statistical analysis}

Statistical analyses were performed using statistical software package SAS Enterprise Guide 7.1. Variables with a normal distribution are presented as means followed by standard deviation (SD) with range values. Correlations of itch intensity with the extent of disease were evaluated by Spearman correlation coefficients. Value of $p<0.05$ was considered significant.

\section{Results}

The mean result of BPDAI was $56.5 \pm 36.2$ (range: 3-148). Pruritus occurring at least once a day was re- ported in $78.1 \%$ (25/32) of BP patients. The presence of pruritus "within the last 3 days" was declared by $93.8 \%$ (30/32) of BP patients. Pruritus tended to persist for more than 10 min (long itch episodes) in $56.3 \%$ of responders (18/32). Antipruritic treatment was used in 53.1\% (17/32) of BP patients (Table 1). Patients with BP reported pruritus more frequently in the evening (72\% of subjects) and at night (62\% of subjects) (Table 2 ). Pruritus aggravating factors in BP patients involved sweating (in 50.0\% of patients), heat (in 59.4\%) and stress (in 46.9\%) (Figure 1). However, BP patients did not observe that physical activities, food and dryness affected pruritus. Pruritus was relieved by cold (34.4\%). The intensity of itch in BP patients expressed by the mean VAS score was $5.8 \pm 2.7$ (range: $0-10$ ) points. The result above 7 points in VAS (denoting at least severe itch) was reported by $22.6 \%$ of patients with BP. The VAS score did not correlate with BPDAI ( $r=$ $-0.04, p=0.83$ ).

Patients declared that itching disturbed falling asleep (53.1\% of respondents) (Table 3 ). Patients were awake at night because of pruritus in $50 \%$ of cases. However, sleeping medications were not used much in BP patients. Patients felt depressed in $59.4 \%$ and reported difficulty in concentrating in $31.3 \%$ because of pruritus.

\section{Discussion}

Despite a great variability of clinical BP manifestations, pruritus represents the leading clinical symptom. To the best of our knowledge, we are presenting the first characteristics of itch in newly diagnosed BP patients based on the itch questionnaire. The study confirmed the clinical observation that pruritus in BP is one of extremely troublesome symptoms to patients. We showed that almost $80 \%$ of patients with BP declared pruritus at least once a day whereas more than $90 \%$ reported pruritus over the previous 3 days. The comparison to literature data obtained in patients with other pruritic diseases such as AD and PV showed that the frequency of daily pruritus in BP patients was between AD and PV. Such declaration was observed in $83-91 \%$ of $A D$ respondents and in $60-90 \%$ of patients with psoriasis [8, 13-19]. In the research conducted by O'Neill et al., the comparison between AD and PV showed more frequent and more intense itch in patients with $A D$ than in patients with PV [20].

In our study, patients with BP presented with pruritus of moderate intensity according to the interpretation of the VAS score. These findings support the data of the recently published study in $\mathrm{BP}$, however the intensity of pruritus in the BP group was assessed as severe, rated at 7.7 in the numeric rating scale (a variant of VAS) [10]. Of note, in the literature, the average itch intensity in $A D$ was noted as moderate, severe or very severe whereas in PV mainly as moderate itch [8, 14, 15, 17, 19]. It also points that pruritus in BP is less intense than in $A D$ and more intense than in PV. 
Table 1. Pruritus characteristics in patients with bullous pemphigoid

\begin{tabular}{lc}
\hline Variable & $\begin{array}{c}\text { Bullous } \\
\text { pemphigoid } \\
n=32(\%)\end{array}$ \\
\hline Daily occurrence of pruritus & $25(78.1 \%)$ \\
\hline Occurrence of pruritus over the previous 3 days & $30(93.8 \%)$ \\
\hline Duration of pruritus episodes > 10 min & $18(56.3 \%)$ \\
\hline Antipruritic treatment & $17(53.1 \%)$ \\
\hline
\end{tabular}

Table 2. Prevalence of pruritus depending on the time of the day in patients with bullous pemphigoid

\begin{tabular}{lcccc}
\hline \multirow{2}{*}{ Answers } & \multicolumn{4}{c}{ Time of the day } \\
\cline { 2 - 5 } & Morning & Afternoon & Evening & Night \\
\hline All time & $7(21.9 \%)$ & $8(25.0 \%)$ & $11(34.4 \%)$ & $12(37.5 \%)$ \\
\hline Often & $7(21.9 \%)$ & $6(18.8 \%)$ & $12(37.5 \%)$ & $8(25.0 \%)$ \\
\hline Seldom & $13(40.6 \%)$ & $14(43.8 \%)$ & $7(21.9 \%)$ & $7(21.9 \%)$ \\
\hline Never & $5(15.6 \%)$ & $4(12.5 \%)$ & $2(6.3 \%)$ & $5(15.6 \%)$ \\
\hline
\end{tabular}

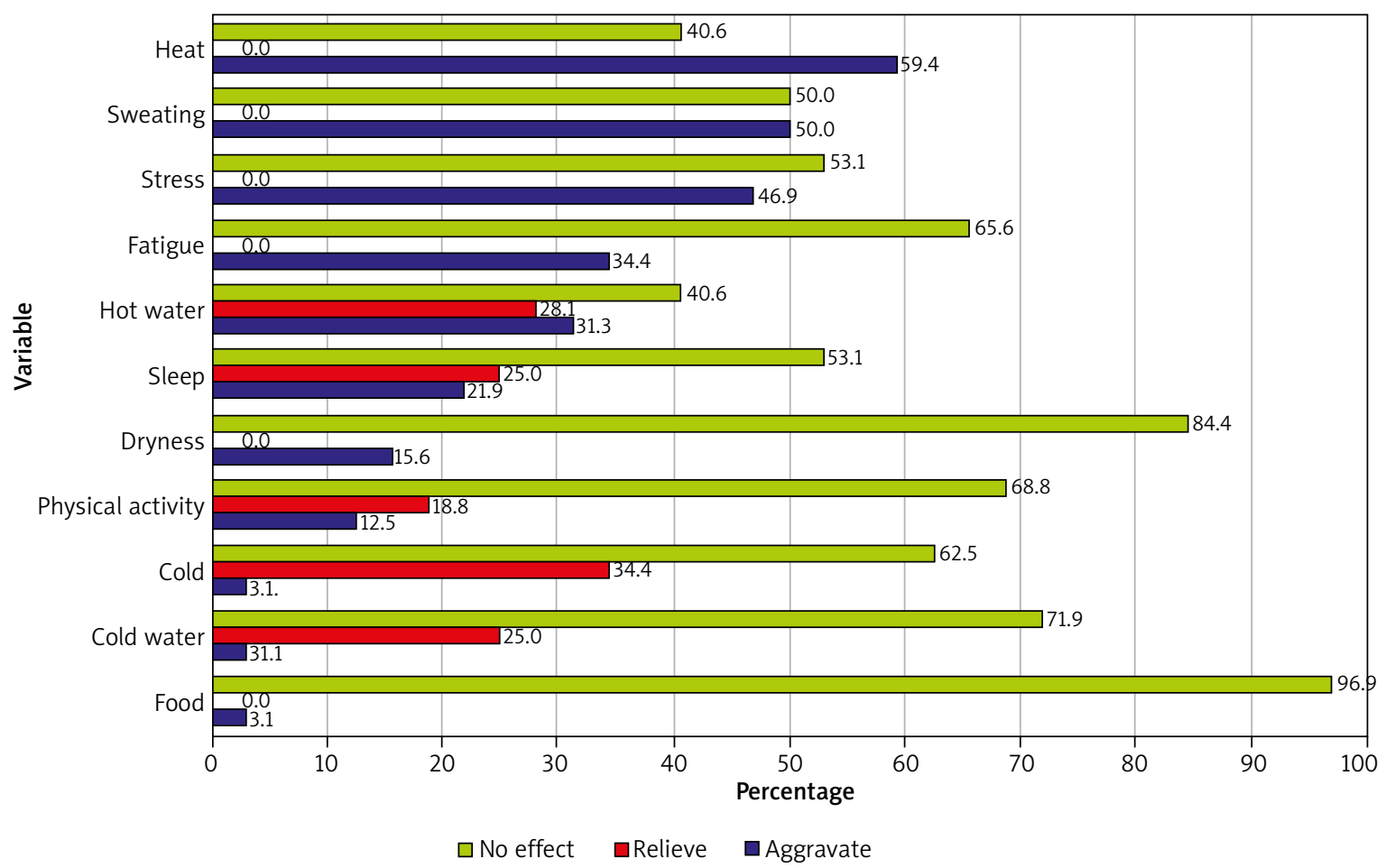

Figure 1. The influence of additional variables on the pruritus occurrence in patients with bullous pemphigoid

During analysis of the relationship between the intensity of pruritus in BP and the extent of skin lesions measured by BPDAI no correlations between the analysed parameters were observed. The same results were presented by Kulczycka-Siennicka et al. who studied the group of 28 patients with BP [10]. Thus, our and the previ-

Table 3. The assessment of sleep quality in patients with bullous pemphigoid

\begin{tabular}{lccc}
\hline Answers & \multicolumn{3}{c}{ Sleep quality } \\
\cline { 2 - 4 } & $\begin{array}{c}\text { Difficulty } \\
\text { falling asleep }\end{array}$ & $\begin{array}{c}\text { Waking up } \\
\text { from sleep }\end{array}$ & $\begin{array}{c}\text { Use of } \\
\text { sleeping } \\
\text { medications }\end{array}$ \\
\hline Almost always & $17(53.1 \%)$ & $16(50.0 \%)$ & $5(16.6 \%)$ \\
\hline Seldom & $4(12.5 \%)$ & $6(18.8 \%)$ & $8(25.0 \%)$ \\
\hline Never & $11(34.4 \%)$ & $10(31.3 \%)$ & $19(59.4 \%)$ \\
\hline
\end{tabular}

ous study indicate that pruritus in BP is an independent symptom from disease severity. However, more studies are required to confirm this observation.

It is interesting to note that more than half of BP patients had long-term episodes of pruritus (lasting longer than 10 min). The majority of surveyed BP patients in the current study reported pruritus in the evening and at night while it was much reduced in the morning when patients were rested. Such sensation of more frequent itch in the evening and at night was reported in patients with $A D$ in the previous studies $[8,13,14]$. The explanation of the above-mentioned observation may be the assertion that pruritus often appears due to tiredness in the evening and/or decreased pruritus threshold in the warmth in bed. Additionally, it is suggested that during the evening rest, patients become more aware of pruritic feelings compared to other day times when they are more active [14]. 
The prevalence of pruritus during evening and night periods should also not be surprising as many patients with itchy diseases often complain about sleep disturbances. In our study, the majority of patients with BP declared that itching impaired their ability to fall asleep and caused awakening from sleep. These findings seem to be underdiagnosed in clinical practice in BP patients. It seems that patients with BP have a higher risk of problems with falling asleep than patients with $A D$ and PV. It was estimated in the literature that $42-84 \%$ responders with $\mathrm{AD}$ and about $70 \%$ patients with psoriasis had problems with falling asleep [8, 9, 14, 15].

In the present study, majority of BP patients considered pruritus as a factor contributing to depression. Of note, bullous pemphigoid is an itching disease but the association with major depressive disorders has also been documented recently [6, 7]. On the other hand, it should be remembered that patients with $\mathrm{BP}$ are elderly and the depression observed in these patients may result from age and other related factors, such as loneliness.

Many factors influence the frequency and intensity of pruritus $[8,14,15,19]$. In the present study, sweating (50.0\%), heat (59.4\%) and stress (46.9\%) were the three most important exacerbating factors in the group of BP patients. Interestingly, there was no clear aggravation due to skin dryness, food, physical activities and hot water. Pruritus in BP was not clearly reduced by any of the assessed factors except for cold.

The exact pathogenesis of pruritus in $\mathrm{BP}$ remains unknown. However, it is suggested that pruritus in BP may be linked to the autoimmune response initiated by loss of self-tolerance against cutaneous autoantigens resulting in the induction of IgG autoantibodies [21]. In fact, 70-96\% of all BP sera contain IgG autoantibodies against BP180 NC16A and their levels correlate with disease activity [21]. Interestingly, some studies disclosed the positive correlation between serum levels of IgG BP180 and BP230 antibodies and intensity of pruritus in BP patients suggesting the role of BP180 as a tool of pruritus evaluation in BP [22, 23]. In addition, recent studies showed that IgG autoantibodies to BP180 and BP230 were also detected in patients with various pruritic disorders who did not yet fulfil all the diagnostic criteria of BP [3, 24]. In these individuals, tissue-bound IgG antibodies were not detected at the epidermal basement membrane. Such patients are suggested to represent a pre-disease state of BP. It is also suggested that itching might lead to the release of a mixture of intracellular BP230 and extracellular components of BP180. Additionally, IgG BP230 autoantibodies are able to induce pro-inflammatory effects such as granulocyte influx triggering pruritus induction followed by the development of anti-BP180 lgG which is named epitope spreading [25]. Concomitant induction of anti-BP180 and anti-BP230 IgG may directly cause itch as a prodromal symptom of $\mathrm{BP}$.
While IgG autoantibodies clearly dominate the autoimmune response in $\mathrm{BP}$, it seems that IgE antibodies might also play a crucial role in the explanation of pathological mechanisms of itchiness in BP [26]. It was showed that IgE antibodies specific for BP180 or BP230 have been detected in clinically active BP [27]. Additionally, it has been shown that the presence of pathogenic BP IgE antibodies contribute to separate the dermal-epidermal adhesion in experimental animal models. Fairley et al. showed that injection of circulating anti-NC16a IgE into a human graft on mice reproduced urticarial lesions [26]. It is hypothesized that circulating and tissue-bound IgE autoantibodies play a role in the initial phases of BP such as urticarial lesions.

The limitation of this study is the small sample representation of BP patients resulting from the evaluation by a single university centre. We believe that our results are important because there are no data on the clinical manifestation of pruritus symptoms in bullous pemphigoid.

\section{Conclusions}

Our study confirms that pruritus is one of the main disturbing symptoms in patients with BP. Physicians should be aware of high frequency, intensity and nature of pruritus in BP. Identification of characteristic sensations associated with pruritus in BP should be included in the therapeutic process of BP patients. On the basis of our experience and study results we would like to recommend introducing antihistaminic drugs in patients with BP and associated pruritus. It is also significant to educate patients about hygiene procedures and the necessity to avoid factors which may exacerbate pruritus.

\section{Acknowledgments}

This work was supported by a grant of the National Centre of Science, Poland, no. N N402 661940.

\section{Conflict of interest}

The authors declare no conflict of interest.

\section{References}

1. Schmidt E, Zillikens D. Pemphigoid diseases. Lancet 2013; 381: 320-32.

2. Murrell DF, Daniel BS, Joly P, et al. Definitions and outcome measures for bullous pemphigoid: recommendations by an international panel of experts. J Am Acad Dermatol 2012; 66: 479-85.

3. Bakker CV, Terra JB, Pas HH, et al. Bullous pemphigoid as pruritus in the elderly: a common presentation. JAMA Dermatol 2013; 149: 950-3.

4. Nakatani T, Inaoki M, Takehara K. Bullous pemphigoid with a prolonged prodrome. J Dermatol 2008; 35: 433-6.

5. Kalinska-Bienias A, Jakubowska B, Kowalewski C, et al. Measuring of quality of life in autoimmune blistering disorders 
in Poland. Validation of disease - specific Autoimmune Bullous Disease Quality of Life (ABQOL) and the Treatment Autoimmune Bullous Disease Quality of Life (TABQOL) questionnaires. Adv Med Sci 2017; 62: 92-6.

6. Forsti AK, Huilaja L, Schmidt E, et al. Neurological and psychiatric associations in bullous pemphigoid-more than skin deep? Exp Dermatol 2017; 26: 1228-34.

7. Kouris A, Platsidaki E, Christodoulou C, et al. Quality of life, depression, anxiety and loneliness in patients with bullous pemphigoid. A case control study. An Bras Dermatol 2016; 91: 601-3.

8. Yosipovitch G, Goon AT, Wee J, et al. Itch characteristics in Chinese patients with atopic dermatitis using a new questionnaire for the assessment of pruritus. Int J Dermatol 2002; 41: 212-6.

9. Ograczyk A, Miniszewska J, Kepska A, et al. Itch, disease coping strategies and quality of life in psoriasis patients. Adv Dermatol Allergol 2014; 31: 299-304.

10. Kulczycka-Siennicka L, Cynkier A, Waszczykowska E, et al. The role of intereukin-31 in pathogenesis of itch and its intensity in a course of bullous pemphigoid and dermatitis herpetiformis. Biomed Res Int 2017; 2017: 5965492.

11. Szepietowski J, Reich A. Itch. Patomechanism, clinics, treatment. Termedia, Poznan 2010.

12. Reich A, Heisig M, Phan NQ, et al. Visual analogue scale: evaluation of the instrument for the assessment of pruritus. Acta Derm Venereol 2012; 92: 497-501.

13. Dawn A, Papoiu AD, Chan YH, et al. Itch characteristics in atopic dermatitis: results of a web-based questionnaire. Br J Dermatol 2009; 160: 642-4.

14. Chrostowska-Plak D, Salomon J, Reich A, et al. Clinical aspects of itch in adult atopic dermatitis patients. Acta Derm Venereol 2009; 89: 379-83.

15. Yosipovitch G, Goon A, Wee J, et al. The prevalence and clinical characteristics of pruritus among patients with extensive psoriasis. Br J Dermatol 2000; 143: 969-73.

16. Reich A, Hrehorow E, Szepietowski JC. Pruritus is an important factor negatively influencing the well-being of psoriatic patients. Acta Derm Venereol 2010; 90: 257-63.

17. Szepietowski JC, Reich A. Pruritus in psoriasis: an update. Eur J Pain 2016; 20: 41-6.

18. Gupta MA, Gupta AK, Kirkby S, et al. Pruritus in psoriasis A prospective study of some psychiatric and dermatologic correlates. Arch Dermatol 1988; 124: 1052-7.

19. Amatya B, Wennersten G, Nordlind K. Patients' perspective of pruritus in chronic plaque psoriasis: a questionnairebased study. J Eur Acad Dermatol Venereol 2008; 22: 822-6.

20. O’Neill JL, Chan YH, Rapp SR, et al. Differences in itch characteristics between psoriasis and atopic dermatitis patients: results of a web-based questionnaire. Acta Derm Venereol 2011; 91: 537-40.

21. Schmidt T, Sitaru C, Amber K, et al. BP180- and BP230-specific IgG autoantibodies in pruritic disorders of the elderly: a preclinical stage of bullous pemphigoid? Br J Dermatol 2014; 171: 212-9.

22. Bardazzi F, Barisani A, Magnano M, et al. Autoantibody serum levels and intensity of pruritus in bullous pemphigoid. Eur J Dermatol 2016; 26: 390-1.

23. Patsatsi A, Kyriakou A, Pavlitou-Tsiontsi A, et al. Association of autoantibodies to BP180 with disease activity in Greek patients with bullous pemphigoid. Clin Dev Immunol 2012; 2012: 854795.

24. Feliciani C, Caldarola G, Kneisel A, et al. IgG autoantibody reactivity against bullous pemphigoid (BP) 180 and BP230 in elderly patients with pruritic dermatoses. $\mathrm{Br}$ J Dermatol 2009; 161: 306-12.

25. Di Zenzo G, Thoma-Uszynski S, Calabresi V, et al. Demonstration of epitope-spreading phenomena in bullous pemphigoid: results of a prospective multicenter study. J Invest Dermatol 2011; 131: 2271-80.

26. Fairley JA, Burnett CT, Fu CL, et al. A pathogenic role for IgE in autoimmunity: bullous pemphigoid IgE reproduces the early phase of lesion development in human skin grafted to nu/ nu mice. J Invest Dermatol 2007; 127: 2605-11.

27. Kalowska M, Ciepiela O, Kowalewski C, et al. Enzyme-linked immunoassay index for anti-NC16a IgG and IgE auto-antibodies correlates with severity and activity of bullous pemphigoid. Acta Derm Venereol 2016; 96: 191-6. 